\title{
THE SYSTEM VANADIUM-SELENIUM NEAR THE COMPOSITION VSe
}

\author{
F. M. A. CARPay \\ Laboratory of Crystal Chemistry, University of Utrecht, The Netherlands
}

(Received 21 April 1966)

\begin{abstract}
Compositions $\mathrm{VSe}_{x}(x=0 \cdot 90-1 \cdot 13)$ were prepared directly by heating the elements. Three series with different temperature treatments were examined. Samples quenched from $1000^{\circ} \mathrm{C}$ all appeared to have only one phase with a NiAs-type structure. The series, cooled very slowly, had two one-phase-regions with NiAs-type structure, namely "VSe" between $\mathrm{VSe}_{0.98}$ and $\mathrm{VSe}_{1.02}$ with $a=3.697 \AA$ and $c=5.990 \AA$ and " $\mathrm{V}_{29} \mathrm{Se}_{32}$ " between $\mathrm{VSe}_{1.10}$ and $\mathrm{VSe}_{1.11}$ with $a=3.606 \AA$ and $c=6.001 \AA$. The latter phase exhibited a superstructure with $a^{\prime}=2 a$ of the NiAs-cell and $c^{\prime}=4 c$ of the NiAs-cell $\left(a^{\prime}=7.211 \AA\right.$ and $\left.c^{\prime}=24.002 \AA\right)$.
\end{abstract}

\section{INTRODUCTION}

THE SYSTEM vanadium-selenium was examined by HoSCHEK and KLEMM ${ }^{(\mathrm{l})}$ and by RøsT and GJERTSEN. ${ }^{(2)}$ HoscheK and KLEMM found in their samples two phases with hexagonal structure: the first one with NiAs-type structure between $\mathrm{VSe}_{1.04}$ and $\mathrm{VSe}_{1 \cdot 13}$ (Figs. 1 and 2) and the second phase with $\mathrm{Cd}(\mathrm{OH})_{2}$-type structure between $\mathrm{VSe}_{1 \cdot 62}$ and $\mathrm{VSe}_{1 \cdot 97}$. TsuBOKAWA ${ }^{(3)}$ who studied the magnetic properties of vanadium sulphide and vanadium selenide in 1959, examined his samples by X-ray techniques and found for VSe a NiAs-type structure (Figs. 1 and 2). RøST and GJERTSEN looked into the whole range of vanadium-selenium compounds prepared with different temperature treatments. They found a tetragonal structure for $\mathrm{V}_{5} \mathrm{Se}_{4}$, except when they quenched from $1000^{\circ} \mathrm{C}$. In all cases there was a homogeneity range extending from the hexagonal NiAs-type structure (Figs. 1 and 2) to a $\mathrm{Cd}(\mathrm{OH})_{2}$-like structure, except for the fact that the slowly-cooled samples had a second phase between $\mathrm{VSe}_{1 \cdot 60}$ and $\mathrm{VSe}_{1 \cdot 80}$. The vanadium-rich boundary of the homogeneity range appeared to be between $\mathrm{VSe}_{0.90}$ and $\mathrm{VSe}_{0.95}$, the selenium-rich boundary near the composition $\mathrm{VSe}_{1.96}$. Between $\mathrm{VSe}_{1 \cdot 12}$ and $\mathrm{VSe}_{1 \cdot 70}$ there was a monoclinic deformation of the structure.

Within the framework of our investigation of the monochalcogenides of the $3 d$-transition metals, we studied the vanadium-selenium compounds, their occurrence, their phase regions, and the physical properties closely bound up with those phases, in all compositions between $\mathrm{VSe}_{0.90}$ (vanadium-rich boundary) and $\mathrm{VSe}_{1 \cdot 13}$ (seleniumrich boundary). The samples were prepared in three different ways and their structures determined accurately by $\mathrm{X}$-ray diffraction.

\section{EXPERIMENTAL}

All samples were prepared directly from the elements by heating $(99.9$ per cent vanadium and 99.999 per cent selenium). The vanadium was powdered in the following way: granules were

(1) E. HoscheK and W. KLemm, $Z$. anorg. allg. Chem. 242, 49 (1939).

(2) E. RøsT and L. GJERTSEN, Z. anorg. allg. Chem. 328, 299 (1964).

(a) I. TsubOKAWA, J. phys. Soc. Japan 14, 196 (1959). 


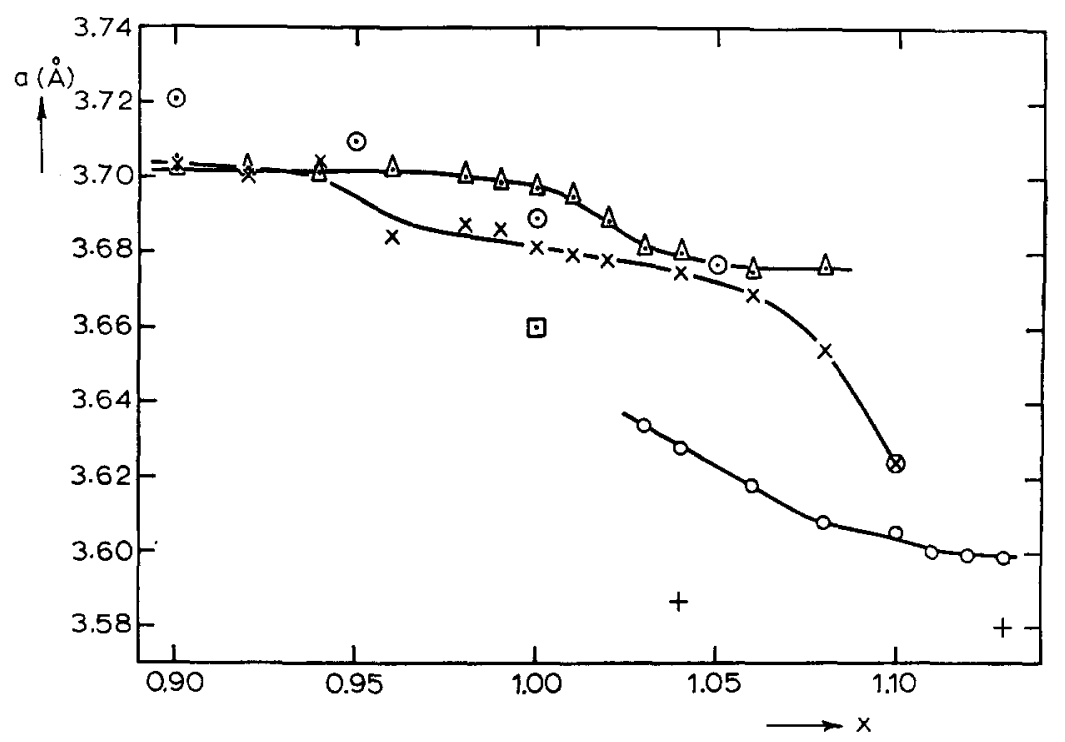

Fig. 1. - Lattice constant $a$ vs. composition of the samples VSe

$\triangle$ phase VSe of slowly-cooled series (III);

$O$ phase $\mathrm{V}_{29} \mathrm{Se}_{32}$ of slowly-cooled series (III);

$\times$ quenched samples (series I);

+ results of HosCHEK and KLEMM;(1)

$\odot$ results of Røst and GJERTSEN;(2)

$\square$ results of TSUBOKAWA. ${ }^{(3)}$

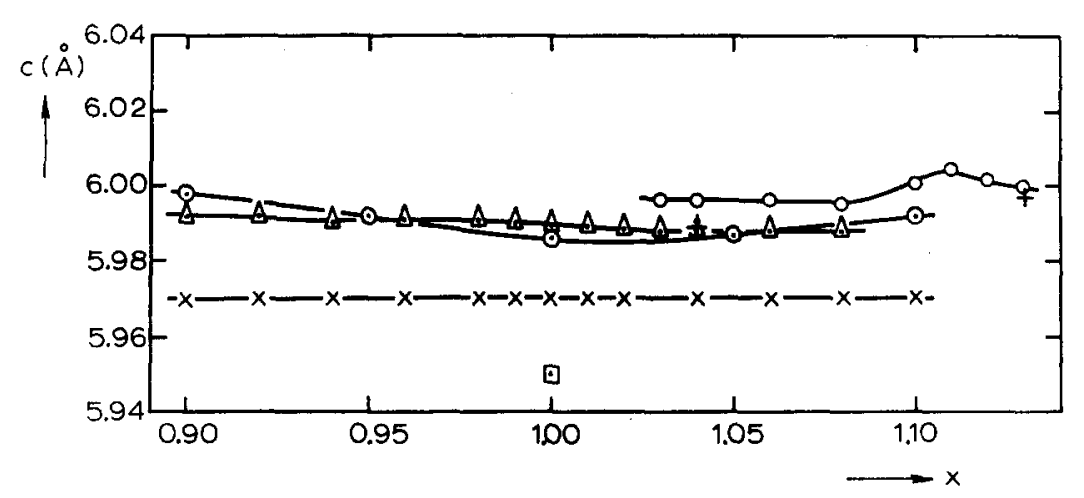

FIG. 2.-Lattice constant $c$ vs. composition of the samples VSe.

saturated with pure $\mathrm{H}_{2}$ and powdered (not very finely). Then the $\mathrm{H}_{\mathrm{z}}$ was extracted by heating the vanadium in a silica tube at $1000^{\circ} \mathrm{C}$ at a final pressure of $10^{-6-10^{-7}} \mathrm{~mm} \mathrm{Hg}$. After this operation the tube was sealed.

The first series. Calculated amounts of powdered vanadium and selenium were weighed out exactly, mixed and put into silica tubes. These tubes were evacuated and heated in a furnace for two days at $800^{\circ} \mathrm{C}$. After opening the tube the sample was powdered again and reheated in an evacuated silica ampulla at $1000^{\circ} \mathrm{C}$. After 7-10 days the ampulla was quenched and the powders were examined by X-ray method.

The second series. The samples were prepared in the same way but the furnace was cooled slowly 
$\left(20^{\circ}\right.$ per $\left.\mathrm{hr}\right)$ after the second heating. Especially in the vanadium-rich compositions the silica was attacked and the samples appeared to contain $\mathrm{V}_{3} \mathrm{Si}$.

The third series. Calculated amounts of powdered vanadium and selenium were mixed, pressed into pellets and put into a silica tube coated with graphite so as to avoid reactions with the silica. The ampulla was evacuated and heated in a furnace at $800^{\circ} \mathrm{C}$ for two days. Then the ampulla was opened, the sample powdered, pressed again into a pellet with a twelve-ton pressure, and heated again in a coated silica tube at $800^{\circ} \mathrm{C}$. After two weeks the furnace was cooled very slowly $\left(10^{\circ}\right.$ per $\mathrm{hr}$ ) and tempered for three days at $600^{\circ} \mathrm{C}$, two days at $450^{\circ} \mathrm{C}$ and again two days at $300^{\circ} \mathrm{C}$. In none of the cases had graphite film and silica been attacked. The pellets were powdered again and examined with X-ray techniques. This third series was studied the most extensively.

The various treatments from opening the tube with powdered vanadium to the evacuation of the tubes with mixed vanadium and selenium did not take more than about $30 \mathrm{~min}$. No noticeable change of the lattice parameters was observed, if these treatments and the re-powdering of the vanadium selenium between the two stages of heating were carried out in a glove box with $\mathrm{N}_{2}$. All X-ray diagrams were made with a Philips diffractometer, using $\mathrm{CuK} \alpha$-radiation.

\section{RESULTS}

The first series. In these samples we found only one phase; the structure is the hexagonal NiAs-type structure. The lattice constant $a$ changes strongly going from $\mathrm{VSe}_{0.90}$ to $\mathrm{VSe}_{1 \cdot 10}$ (Fig. 1) the constant $c$ remaining the same or nearly so (Fig. 2). Superstructures were not found. As $\mathrm{V}_{3} \mathrm{Si}$, and probably $\mathrm{V}_{5} \mathrm{Se}_{4}$, appeared at the vanadium-rich boundary, difficulties arose in the determination of the composition of the VSe-phase in these samples.

The second series. In this series clearly different phases appeared. The $\mathrm{V}_{\mathbf{3}} \mathrm{Si}$ disturbed especially at the vanadium-rich boundary. In the third series there was no attack of the silica tube; the further results were like those of the second series, that is, the same phases and the same lattice constants, so we shall not deal with the second series here (maybe the character of the vanadium-rich side of the range is somewhat different from that of the third series, notably at the point of the $\mathrm{V}_{5} \mathrm{Se}_{4}$-phase; see the discussion).

The third series. (Figs. 1 and 2). This slowly cooled series was studied the most extensively. There are in the range $\mathrm{VSe}_{x}(x=0.90-1 \cdot 13)$ two one-phase regions: the phase "VSe" with a broad region of existence near $x=1.00$ (with $a=3.697 \AA$ and $c=5.990 \AA$ ) and the phase " $\mathrm{V}_{29} \mathrm{Se}_{32}$ " with a narrow region of existence between $x=1.10$ and 1.11 (with $a=3.606 \AA$ and $c=6.001 \AA$, $a$ and $c$ being the lattice constants of the simple NiAs-cell). $\mathrm{VSe}_{\mathbf{0}} \cdot 90$ shows in addition to the main constituent VSe, weaker reflections in the X-ray diagram due to the tetragonal phase $\mathrm{V}_{5} \mathrm{Se}_{4}$ of RøST and GJERTSEN. ${ }^{(2)}$ The lattice constants are $a=9.27 \AA$ and $c=3.41 \AA$. The $\mathrm{X}$-ray diagrams of $\mathrm{VSe}_{\mathbf{0}^{.92}}$ and $\mathrm{VSe}_{\mathbf{0 . 9 4}}$ are also two-phase diagrams: VSe besides $\mathrm{V}_{5} \mathrm{Se}_{4}$. At VSe ${ }_{0.96}$ the reflections of $\mathrm{V}_{5} \mathrm{Se}_{4}$ were present but too weak to be measured.

The X-ray diagrams of $\mathrm{VSe}_{0.98}-\mathrm{VSe}_{1.02}$ show only the VSe-phase with NiAsstructure. In this region the lattice constant $a$ changes with the composition, the lattice constant $c$ remaining nearly the same. At $\mathrm{VSe}_{1.03}$ a new phase appears besides $\mathrm{VSe}$, namely $\mathrm{V}_{29} \mathrm{Se}_{32}$. Between $\mathrm{VSe}_{1 \cdot 03}$ and $\mathrm{VSe}_{1 \cdot 10}$ only these two phases occur, proportionate to the composition. In the two-phase regions below $\mathrm{VSe}_{0.88}$ and above $\mathrm{VSe}_{1.02}$ the lattice parameters $a$ and $c$ of the VSe-phase remain almost the same. $\mathrm{VSe}_{1 \cdot 10}$ and $\mathrm{VSe}_{1 \cdot 11}$ both give $\mathrm{V}_{29} \mathrm{Se}_{32}\left(\mathrm{VSe}_{1 \cdot 104}\right)$, at $\mathrm{VSe}_{1 \cdot 10}$ the strongest reflections of VSe being very faint and at $\mathrm{VSe}_{1 \cdot 11}$ a new phase appearing to be very faint too. Consequently the homogeneity region of $\mathrm{V}_{29} \mathrm{Se}_{32}$ is very narrow, lying between $\mathrm{VSe}_{1 \cdot 10}$ 
and $\mathrm{VSe}_{1 \cdot 11}$. The structure is that of the NiAs-type with lattice constants different from the parameters of VSe. The $a$ changes a little within the narrow one-phase region, $c$ remaining almost the same. At $\mathrm{VSe}_{x}$ with $x>1.11$ parameters $a$ and $c$ remain nearly the same; with $x<1.10 a$ changes in a remarkable way, $c$ being constant. The decrease of $a$ with increasing $x$ is not quite clear, because a two-phase region is concerned. This may be the result of a still too rapid cooling. In addition

\begin{tabular}{|c|c|c|c|}
\hline$h k l$ & $d(\exp )$ & $d$ (calc) & $I_{\mathrm{obs}}$ \\
\hline \multirow[t]{2}{*}{101} & & 6.04 & \\
\hline & 6.02 & & 2 \\
\hline 102 & $5 \cdot 54$ & $5 \cdot 54$ & 1 \\
\hline 103 & 4.92 & 4.92 & 1 \\
\hline 105 & $3 \cdot 80$ & $3 \cdot 81$ & 1 \\
\hline 111 & $3 \cdot 57$ & $3 \cdot 57$ & 1 \\
\hline 112 & 3.45 & 3.45 & $<1$ \\
\hline 106 & $3 \cdot 37$ & $3 \cdot 37$ & $<1$ \\
\hline 113 & $3 \cdot 29$ & $3 \cdot 29$ & 1 \\
\hline $200(100)$ & $3 \cdot 12$ & $3 \cdot 12$ & $<1$ \\
\hline $008(002)$ & 2.999 & 3.002 & 7 \\
\hline 204 (101) & $2 \cdot 773$ & $2 \cdot 770$ & 100 \\
\hline 116 & 2.674 & 2.679 & 1 \\
\hline 117 & $2 \cdot 485$ & $2 \cdot 486$ & 1.5 \\
\hline 109 & $2 \cdot 451$ & $2 \cdot 454$ & $<1$ \\
\hline 0010 & $2 \cdot 396$ & 2.402 & $\ll 1$ \\
\hline 211 & $2 \cdot 348$ & $2 \cdot 349$ & $<1$ \\
\hline 212 & $2 \cdot 314$ & $2 \cdot 316$ & 1 \\
\hline 213 & $2 \cdot 263$ & $2 \cdot 264$ & 1.5 \\
\hline 1010 & $2 \cdot 238$ & $2 \cdot 242$ & $<1$ \\
\hline $208(102)$ & $2 \cdot 164$ & $2 \cdot 164$ & 82 \\
\hline 215 & $2 \cdot 118$ & $2 \cdot 118$ & 2 \\
\hline 301 & $2 \cdot 074$ & 2.074 & $\ll 1$ \\
\hline $302 ?$ & 2.057 & 2.051 & 1 \\
\hline 216 & $2 \cdot 033$ & $2 \cdot 033$ & $\ll 1$ \\
\hline 303 & $2 \cdot 014$ & $2 \cdot 015$ & $<1$ \\
\hline 305 & 1.908 & 1.910 & $<1$ \\
\hline
\end{tabular}

the lattice has a superstructure, with $a^{\prime}=2 a$ and $c^{\prime}=4 c$ ( $a^{\prime}$ and $c^{\prime}$ being the lattice parameters of the supercell, $a$ and $c$ of the NiAs-cell). The superstructure reflections are faint, but clear; see Table 1 . The indices $h k l$ are according to the supercell; in parentheses the $h k l$ 's of the NiAs-cell.

$\mathrm{VSe}_{1 \cdot 11}, \mathrm{VSe}_{1 \cdot 12}$ and $\mathrm{VSe}_{1 \cdot 13}$ all consist of the phase $\mathrm{V}_{29} \mathrm{Se}_{32}$ together with the growing reflections of a new phase, probably the monoclinic $\mathrm{V}_{3} \mathrm{Se}_{4} \cdot{ }^{(2)} \mathrm{We}$ did not investigate this further.

Finally it must be noticed that in none of the samples vanadium- or seleniumreflections were measured. 


\section{DISCUSSION}

The tetragonal phase $\mathrm{V}_{5} \mathrm{Se}_{4}$ is the most explicit in the slowly-cooled $\mathrm{VSe}_{0.90}$, but much less than would be expected from the composition of the sample (about 50 per cent $\mathrm{V}_{5} \mathrm{Se}_{4}$ and about 50 per cent VSe). The lattice constant $a$ of the VSe-phase in $\mathrm{VSe}_{\mathbf{0}} \cdot 90$ is larger than in $\mathrm{VSe}_{\mathbf{0} 98}$ which supports the supposition that the mixedcrystal region extends-at the vanadium-rich side-still further than $\mathrm{VSe}_{0.98}$. In the second and first series too there are weak $\mathrm{V}_{5} \mathrm{Se}_{4}$ reflections in $\mathrm{VSe}_{0 \cdot 90}$. This is in contrast with the results of Røst and GJERTSEN. ${ }^{(2)}$ In their experiments there are no reflections of $\mathrm{V}_{5} \mathrm{Se}_{4}$ in samples quenched from $1000^{\circ} \mathrm{C}$. That the reflections of the first and second series are weaker than of the third may be connected with the presence of $\mathrm{V}_{3} \mathrm{Si}$-on account of the high-temperature treatment-which would mean that the vanadium-selenium compound contains less vanadium.

The phase VSe exhibits a pure NiAs-structure without supercell and occurs on both sides of the composition $\mathrm{VSe}_{1 \cdot 00}$. In the case of $\mathrm{VSe}_{1 \cdot 00}$ the axial ratio $c / a=$ 1.620. Vanadium-selenium is one of the few compounds that has a NiAs-structure at the exact atomic ratio $1: 1$.

The phase $\mathrm{V}_{29} \mathrm{Se}_{32}$ has a NiAs-type structure with supercell and lattice constants $a^{\prime}=7 \cdot 211 \AA$ and $c^{\prime}=24.002 \AA$. The axial ratio of the NiAs-cell $c / a=1.664$. The position of atoms and vacancies in this superstructure has not yet been determined. The structure of $\mathrm{V}_{29} \mathrm{Se}_{32}$ has not yet been observed in any other similar compound with NiAs-type structure.

If $\mathrm{V}_{29} \mathrm{Se}_{32}$ is a ionic compound, it confirms the theory of BERTAUT ${ }^{(4)}$ that all ionic defect-structures should be ordered.

Further experiments with the system $\mathrm{V}-\mathrm{Se}$ are at this moment in preparation and in progress, and will be published in due time.

Acknowledgements-The author is very grateful to Professor Dr. J. H. van SANTEN for the valuable discussions and for reading the manuscript. He is also indebted to Miss E. DEN DeKKER and Mr. C. LANGEREIS of the Philips Research Laboratories for making the X-ray diagrams and for their assistance at interpreting the results.

(4) E. F. BerTAut, Acta crystallogr. 6, 557 (1953). 